\title{
Experimental Characterization of Optical Fiber Long Period Grating with Reduced Temperature Sensitivity
}

\author{
Tarun Sharma, Himanshu Monga, Vipul Sharma, and Sandip Vijay
}

\begin{abstract}
In this paper we show the experimental characterization of optical fiber long period grating for the reduced temperature sensitivity by optimizing the refractiveindex profile of the host fiber. In Long Period Fiber grating the fundamental guided mode light couple with the into copropagating cladding modes at various wavelengths. The two layer Geometry later on the three layer geometry which is useful for refractive index sensing sensing application. The sensitivity of LPFGs to environmental parameters is influenced by the period of the LPFG by the order of the cladding mode to which coupling takes place and by the composition of the optical fiber. A novel method to compensate the temperature sensitivity using a boron co-doped germanosilicate core fiber is used as a host fiber. The Theoretical Characteristic Results matches with the experimental results which demonstrate that such gratings with reduced temperature coefficient retain their capability to detect strain and ambient index variations.
\end{abstract}

Index Terms-LPFG, FBG, refractive index, temperature sensitivity, cladding Mode, OSA.

\section{INTRODUCTION}

A long-period fiber grating (LPG), which couples light from a fundamental guided core mode into co-propagating cladding modes at various wavelengths, was first reported by Vengsarkar and co-workers in1996 [1]. LPGs have also been used as gain-flattening filters for erbium-doped fiber amplifiers [2], and as optical fiber polarizer's [3].

As in the fiber Bragg grating (FBG), the LPG is sensitive to measured such as temperature or strain, which may alter the period of the grating or the refractive index of the core or cladding. Unlike FBG the cladding mode configuration of the LPG is extremely sensitive to the refractive index of the medium surrounding the cladding, thus allowing it to be used as an ambient index sensor. Compared to other optical sensors, LPGs offer many advantages such as low insertion losses, low back reflection and relatively simple fabrication, but they do show high temperature sensitivity. However, temperature sensitivity can normally be controlled by using a second grating as a control, or by coating the cladding with a suitable material with a refractive index designed to compensate for temperature.

LPGs are transmission gratings in which the coupling is between forward propagating core and cladding modes. Typically in a single mode fiber (see figure 1) an LPG couples the fundamental guided core mode to a co-

Manuscript received May 23, 2011; revised July 13, 2011.

Tarun Sharma is with the Shoolini University, Solan (H.P). ( emailSharma.tarun23@gmail.com.)

Himanshu Monga is with the shoolini university, solan. (H.P).

Vipul Sharma is with the GKV, Haridwar. (U.K)

Dr. Sandip Vijay is with DIT Dehradun. (U.K) propagating cladding mode at a coupling (or resonance) wavelength given by

$$
\lambda_{\mathrm{i}}^{\mathrm{n}}=\left(\mathrm{n}_{\text {eff.co }}(\lambda \mathrm{i})-\mathrm{n}_{\text {eff.cl }}(\lambda \mathrm{i})\right) \Lambda[4]
$$

where $\lambda$ ni is the nth coupling wavelength, neff.co $(\lambda i)$ is the effective index of the core at the wavelength $\lambda i$ and neff.cl $(\lambda i)$ is the effective index of the nth cladding mode at the wavelength $\lambda \mathrm{i}$. $\Lambda$ is the LPG period, which is much longer for co propagating coupling at a given wavelength than for the counter propagating coupling. In this paper we used the boron co doped germanosilicate core fiber as a host fiber to reduce the Temperature sensitivity.

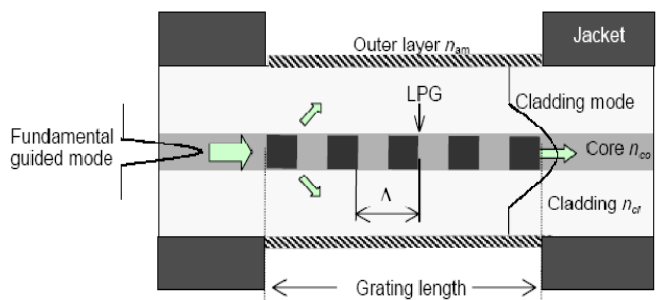

Fig.1. Long Period Grating[4]

\section{CHARACTERIZATION OF THE LPFG AS TEMPERATURE SENSITIVITY}

The sensitivity of LPFGs to environmental parameters is influenced by the period of the LPFG by the order of the cladding mode to which coupling takes place and by the composition of the optical fiber. This combination of influences allows the fabrication of LPFGs that have a range of responses to a particular measured - a single LPFG may have attenuation [5] bands that have a positive sensitivity to a measured, others that are insensitive to the measured and others with a negative sensitivity. This property has been widely exploited for controlling the temperature sensitivity of LPFGs. For many telecommunications applications spectral stability is of prime importance, and the ability to fabricate an LPFG with an inherently temperature insensitive attenuation band is an attractive feature. This is also attractive for forming temperature-insensitive strain sensors. On the other hand, for forming temperature sensors, or thermally tuned filters, a high temperature sensitivity (of either sign) is required. The origin of the temperature sensitivity may be understood by differentiating equation the sensitivity of the LPFG to perturbations in external refractive index is only due to the change in wavelength per change in differential refractive index, as given in equation(2)[6].

$$
\begin{gathered}
\frac{\mathrm{d} \lambda_{0}}{\mathrm{~d}\left(\delta \mathrm{n}_{\text {eff }}\right.}=\frac{\mathrm{d} \lambda_{0}}{\delta \mathrm{n}_{\text {eff }-\delta \mathrm{m}_{\mathrm{g}}}} \\
\frac{\mathrm{d} \lambda}{\mathrm{dT}}=\frac{\mathrm{d} \lambda}{\mathrm{d}(\delta \text { neff })}\left(\frac{\mathrm{dneff}}{\mathrm{dT}}-\frac{\mathrm{dncl}}{\mathrm{dT}}\right)+\Lambda \frac{\mathrm{d} \lambda}{\mathrm{d} \Lambda} \frac{1}{\mathrm{~L}} \frac{\mathrm{Dl}}{\mathrm{Dt}}
\end{gathered}
$$

where $\lambda$ is the central wavelength of the attenuation band, $T$ is the temperature, $n_{\text {eff }}$ is the effective refractive index of 
the core mode, $\mathrm{n}_{\mathrm{cl}}$ is the effective refractive index of the cladding mode, $\delta$ neff $=\left(\mathrm{n}_{\mathrm{eff}}-\mathrm{n}_{\mathrm{cl}}\right)$, $\mathrm{L}$ is the length of the LPFG and $\Lambda$ is the period of the LPFG. The first term on the right-hand side of equation (3) is the material contribution, and is related to the change in the differential refractive index of the core and cladding arising from the thermo-optic effect. This contribution is dependent upon the composition of the fiber and is strongly dependent upon the order of the cladding mode. For coupling to low order cladding modes (accessed using longer periods, $\Lambda>100 \mu \mathrm{m}$ ), the material effect dominates. For coupling to higher-order cladding modes (accessed using shorter periods $<100 \mu \mathrm{m}$ ), the material effect for standard germanosilicate fibers can be negligible. The second term is the waveguide contribution as it results from changes in the LPFG's period. The magnitude and sign of the term depend upon the order of the cladding mode. For coupling to low-order cladding modes $\mathrm{d} \lambda / \mathrm{d} \Lambda$ is positive, while for the higher-order cladding modes this term is negative. Thus, by an appropriate choice of LPFG period it is possible to balance the two contributions to the temperature sensitivity to produce a temperature-independent attenuation band and also to produce attenuation bands with temperature sensitivities (positive or negative) appropriate to specific applications. Altering the fiber composition, such that the thermo-optic coefficient of the core is either larger or smaller than that of the cladding, can also be used to obtain required temperature sensitivity. In addition to the change in central wavelength, a change in the extinction of the attenuation band may be observed for LPFGs with strength $\kappa L>\pi$. At room temperature, the temperature response of the wavelengths of the attenuation bands' is linear, as illustrated in figure (2) LPGs fabricated in standard telecommunications optical fiber exhibit temperature sensitivities in the range $3 \mathrm{~nm} / 100{ }^{\circ} \mathrm{C}$ to $10 \mathrm{~nm} / 100{ }^{\circ} \mathrm{C}$ [5]. This is an order of magnitude larger than the sensitivity of FBG sensors.

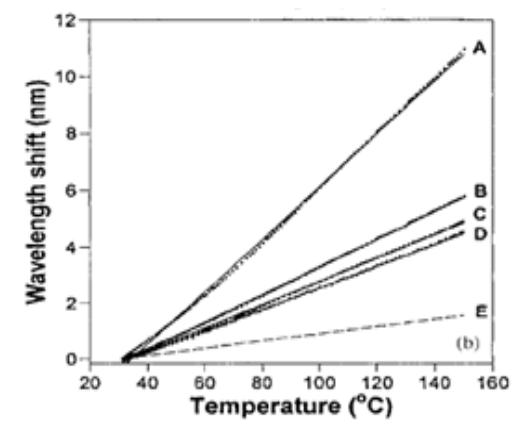

Fig. 2. Shift in the wavelengths of four attenuation bands [21] A-D, as a function of temperature for the LPFG dashed Line shows response at 1550 $\mathrm{nm}$ for the LPFG. Linear response of the Temperature is observed.[7]

\section{EXPERIMENTAL CHARACTERIZATION OF THE LONG} PERIOD FiBER GRATING FOR TEMPERATURE SENSITIVITY

Experiment for Temperature sensitivity is done with Boron- co- doped Fiber. Specification for the Boron co doped Fiber is given below.

\section{TEMPERATURE SENSITIVITY EXPERIMENT}

Our experiment FOR the temperature sensitivity is done with the same fiber parameter at the $1550 \mathrm{~nm}$ wavelength. we obtain the linear temperature response with respect to the wavelength shift.

Table I: Specifications For Designing of LPFG using the Boron-Co- Doped Fiber. To investigate the response of the LPFG For temperature by considering the three layer structure we use single mode fiber.

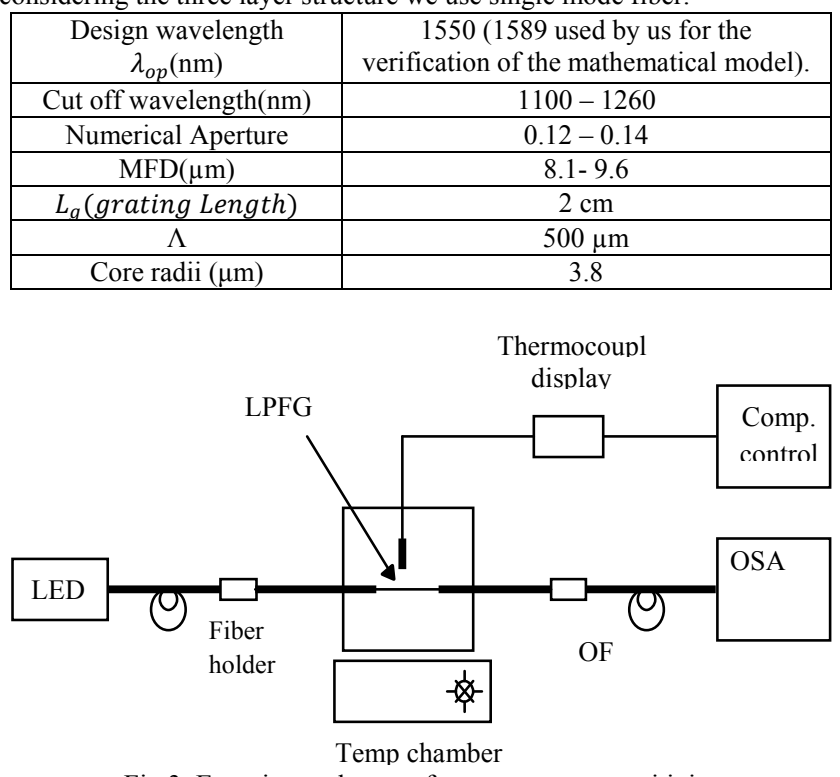

Fig.3. Experimental set up for temperature sensitivity

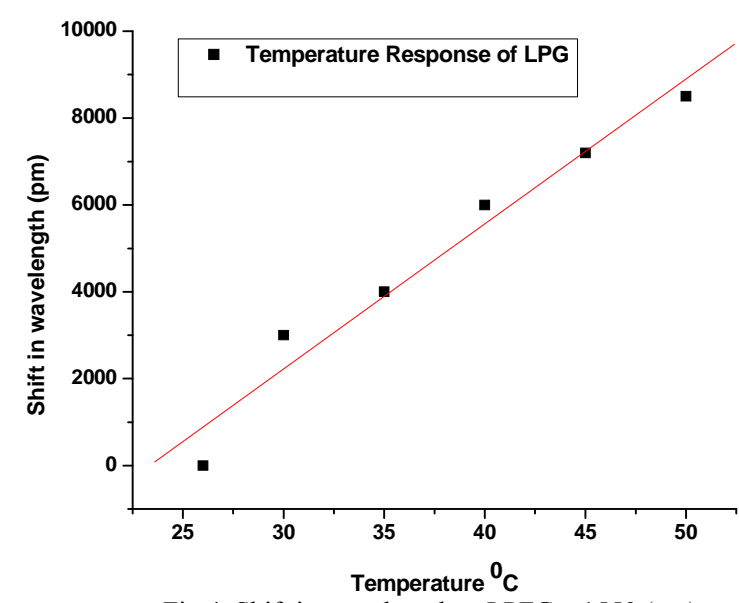

Fig.4. Shift in wavelength at LPFG at1550 (nm)

Table II: Temperature sensitivity of the LPFG

\begin{tabular}{|c|c|}
\hline Temperature & Shift in wavelength $(\mathrm{pm})$ \\
\hline 26 & 0 \\
\hline 30 & 3000 \\
\hline 35 & 4000 \\
\hline 40 & 6000 \\
\hline 55 & 7200 \\
\hline
\end{tabular}

\section{CONCLUSION}

For Temperature sensitivity, we obtain a linear response with respect to the wavelength and temperature. The experiment shows that there is approximate $8 \mathrm{~nm}$ shift in the wavelength for the 50 degree centigrade temperature. Thus the theoretical value matches with the experimental value. We have shown that long-period gratings with reduced temperature sensitivity can be fabricated in commonly available fibers. So, this type of sensor have very good practical applicability in aero plane, space shuttle, One of the fabulous uses for these sensors is to actually provide a 
temperature limit signal for operating jet engines in flying aircraft.

\section{ACKNOWLEDGMENT}

The author is thankful to CSIO, Chandigarh to give their permission to carried out the work. the auther is also thankful to Dr. derick angles, department of electronics technology, gndu Amritsar for his valuable suggestion.

\section{REFERENCES}

[1] Vengsarkar A M, Lemaire P J, Judkins J B, Bhatia V, Erdogan T and Sipe J E 1996 Long-period grating asband-rejection filters . 14 5864.J. Light wave Technol.

[2] Qian J R and Chen H E 1998 Gain flattening fibre filters usingphase shifted long period fibre grating 34 1132-3Electron. Lett. .

[3] [3] Ortega B, Dong I, Lic W E, Sandro J P, Reekie I, Tsypina S I,Bagratashvile V N and Laming R I 1997 High performance.

[4] Optical fiber polarizer's based on long-period gratings in birefringent optical fibers 9 1370-2. IEEE Photonics Technol. Lett. .

[5] R Hou, Z Ghassemlooy, "Modeling of long-period fiber grating response to refractive index higher than that of cladding" institute of physics publishing Meas. 1-5 Sci. Technol. 12 (2001) .

[6] Bhatia V 1999 "Applications of long-period gratings to single and multi-parameter sensing",. 457-66. Opt. Express

[7] James, S.W. \& Tatam, R.P. (2003). "Optical fibre long-period grating sensors: characteristics and applications,". Measurement Science and Technology.

[8] T. Erdogan, "Fibre grating spectra," vol. 15, pp. 1277-1294, 1997IEEE J. Lightwave Technol.

[9] Hannes Hochreiner, Michael Cada, and Peter D. Wentzell, "Modeling the Response of a Long-Period Fiber Grating to Ambient Refractive Index Change in Chemical Sensing Applications", vol. 26, no. 13, July 1, 2008.Journal of Light wave Technology.

[10] Bhatia V, Campbell D, Claus R O and Vengsarkar A M 1997 "Simultaneous strain and temperature measurement with long-period gratings," . 22 648-50. Opt. Letter.

[11] Shu X, Allsop T, Gwandu B, Zhang L and Bennion I 2001 "High temperature sensitivity of long-period gratings in $\mathrm{B}-\mathrm{Ge}$ co doped fiber," 13 818-20.IEEE Photon. Technol. Lett.

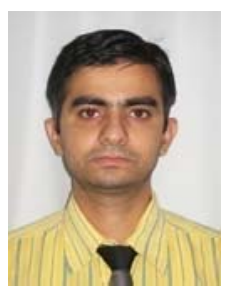

Bibliography Tarun Sharma was Born in Vill. Berly, P.O. Bharoli, The Dehra, Distt Kangra, Himachal Pradesh in 1984. He did his M.Sc( Applied Electronics) From Gurukula Kangri Vishvidyala, hardware in 2006. After that he completed His M.Tech(Communication Systems) From GNDU, Amritsar in 2009. Presently he is working as assistant prof(ECE) IN SCHOOL IN ENGG AND TECHNOLOGY, Shoolini university, solan
(Himachal Pradesh). His area of interest in fiber optic Communication, Fiber optic sensors, Wireless sensors networks. He has more than 3 years of teaching Experience. He has 5 paper in international conference and 2 paper in international Journal.

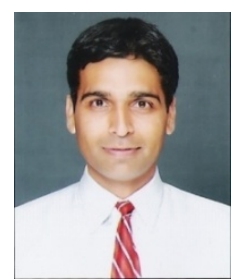

Himanshu Monga Was born in Joginder Nagar, Himachal Pradesh, India, on 06th November 1974. He obtained his bachelor's degree in electronics \& Communication Engineering from Amravati University, Maharastra, India and Master's degree in Electronics and Telecommunication Engineering \& Masters degree in management from IGNOU in Human resource management. $\mathrm{He}$ is pursuing $\mathrm{Ph}$.D.from Thapar Institute of Engineering and Technology, Patiala. Presently, He is working as Associate Professor in Electronics and Communication Engineering department with Shoolini University of Biotechnology \& Management sciences, Solan, Himachal Pradesh. His interests are fiber optics communication, wavelength division multiplexing \& OCDMA. He has 15 Research papers in international/national conferences.

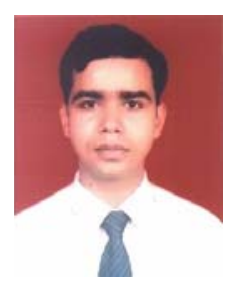

Vipul Sharma was born in Haridwar, Uttrakhand, India, in 1974. He obtained his bachelor's degree in Electronics \& Telecommunication Engineering from North Maharashra University, Maharastra, India and Master's degree in Microwave Engg \& Radar,IIT, Roorkee. He is pursuing Ph.D.from Punjab Technical University, Jalandhar. He is working as Assistant Professor in Electronics and Communication Engineering department with Gurukul Kangri Vishwavidyalaya, Haridwar. His interests are Soft Computing, Antennas and their Biomedical applications. He has 10 Research papers in International/National Journals and 12 papers in International/National conferences.

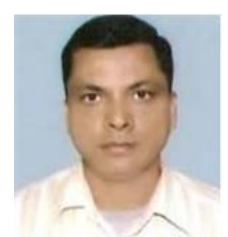

Sandip Vijay received B.Sc. (Engg.) from PIT Patna (erstwhile Magadh University) in 2000 M.Tech. (Electronics \& Comm. Engg.) in 2005, the member of IEEE(USA), NSBE (USA), IANEG(USA), ISOC (USA),Life Member of ISTE (INDIA) has published over Fifty research papers in national and international journals/conferences and IEEE proceeding publication in field of Wireless \& Digital Communication Network. He finished his Doctorate from I.I.T. Roorkee in the field of Wireless Computing under Ministry of HRD, Government of India fellowship. Currently working as a Head of Department and Professor in Department of ECE \& AEI at Dehradun Institute of Technology (DIT) Total Publication Till Date: 81 . 